\title{
Unique Case of Bilateral Exudative Retinal Detachment following Creatine Supplementation in a Patient with Autosomal Dominant Bestrophinopathy
}

\author{
Konstantinos Kopsidas ${ }^{a}$ Hedayat Javidi $^{b}$ Simon P. Kelly ${ }^{c}$ Tariq Aslam ${ }^{a}$ b \\ Graeme Black $^{\mathrm{d}}$ Sajjad Mahmood ${ }^{\mathrm{a}}$ b \\ aManchester Royal Eye Hospital, Manchester University NHS Foundation Trust, Manchester, UK \\ bFaculty of Biology Medicine and Health, University of Manchester, Manchester, UK; \\ 'Department of Ophthalmology, Bolton NHS Foundation Trust, Bolton, UK; \\ dManchester Centre for Genomic Medicine, University of Manchester, Manchester, UK
}

\section{Keywords}

Creatine $\cdot$ Retina $\cdot$ Retinal dystrophy $\cdot$ Retinal detachment $\cdot$ Bestrophinopathy

\section{Abstract}

We report a case of bilateral serous retinal detachment in a patient with rod-cone dystrophy caused by mutation of BEST1. This followed creatine monohydrate use as a dietary supplement. A 39-year-old male with rod-cone dystrophy and low hyperopia developed extensive bilateral exudative retinal detachment following creatine monohydrate diet supplementation. Five days after stopping creatine use, the bilateral retinal detachments resolved completely. This may indicate a causative relation of creatine supplementation to development of serous retinal detachment in a susceptible patient with pre-existing retinal dystrophy. 


\section{Introduction}

Creatine supplementation is in widespread use as a diet supplement. It is a popular supplement among bodybuilders and is available without a doctor's prescription. Our patient used this as part of his exercise regime.

\section{Case Presentation}

A 39-year-old male presented with a 1-week history of blurred vision in both eyes. In the week preceding, he had commenced taking creatine monohydrate, a dietary supplement he had used aspiring to help muscle growth.

He had a family history of pan-retinal rod-cone dystrophy and his case has been documented and reported as missense variant I205T [1]. He was otherwise fit and well and undertaking extensive physical exercise training. His medical history was unremarkable except that he was on mometasone nasal spray for postnasal drip. On presentation, the visual acuity was $6 / 36$ in the right and 6/60 in the left eye. His refraction was low hyperopia (OD +3.75, OS +4.00 ). Fundus examination showed bilateral total exudative retinal detachment. His last recorded visual acuity before this acute presentation was $6 / 18$ in the right eye and 6/36 in the left eye. Extensive investigations including serology for inflammatory, autoimmune, and infectious disease and a chest X-ray were all negative. The patient was monitored using widefield fundus imaging and optical coherence tomography (Fig. 1, Fig. 2). Fluorescein angiography (Fig. 3) showed widespread accumulation of fluorescein related to the serous detachment with no other signs of a vascular event or focal exudative process.

The retinal detachments resolved spontaneously within a week of cessation of creatine supplementation. Visual acuity recovered to $6 / 30$ in the right eye and 6/48 in the left.

\section{Discussion}

Creatine is a dietary supplement claimed to "help muscle growth, endurance, strength, power, and recovery," according to the manufacturers, and it is commonly used by bodybuilders. Before commencing training, a 5-day "loading phase" is recommended, followed by daily supplementation on training days. Our patient's symptoms began on the fourth day of the loading phase. He took the supplement for 5 days and then presented to ophthalmology outpatients. Discontinuing the creatine led to rapid spontaneous resolution.

The mechanism by which creatine caused this problem is unknown. However, creatine and phosphocreatine are essential molecules that maintain ATP levels in high-energy-demanding tissues such as the retina [2]. Creatine is obtained from diet and maintained in cells with a plasma membrane creatine transporter that transports creatine from the blood circulation into the cell. In the human retina, the main sites of creatine transporter localization are the blood retinal barriers, namely the inner retinal blood vessels and the retinal pigment epithelium (RPE), and also the outer retina segments, transporting creatine from the blood circulation towards and into the photoreceptors [3].

Studies have shown that in mammals, creatine is transported in an unchanged form from the blood to the retina across the blood retinal barrier against the creatine concentration gradient that exists between the retina and blood in at least a 2-log-scale order of magnitude, and in a flow rate approximately 20 -fold greater than that of sucrose [4]. Currently, no visual side 
effects from the use of creatine supplementation have been reported; however, there is conflicting evidence of an adverse effect on renal function. Isolated reports of acute tubular necrosis and interstitial nephritis have been associated with dietary creatine use [5].

This patient's underlying retinal dystrophy may have put him at risk when he took the creatine supplements. Photoreceptor integrity is essential for maintaining the anatomical relationship as well as metabolic function of the neuroretina and the RPE. Breakdown of these interconnections can happen in photoreceptor degeneration and lead to anatomical and functional alterations by decompensating the ability of the RPE to pump fluid towards the choroid [6]. The BEST1 gene encodes the Bestrophin-1 protein, which is thought to regulate calciumactivated chloride channels, primarily within the RPE. BEST1 mutations have been associated with a variety of retinal dystrophies [1].

In this patient, a relative reduction of photoreceptors and reduced ability to metabolize creatine may have led to its accumulation in the outer retina layers causing an excessive osmotic gradient and fluid flow towards the subretinal space. Furthermore, we speculate that the osmotic gradient could not be counterbalanced by the patient's decompensated RPE function.

A differential in this patient relates to his use of a steroid mometasone nasally following a recent upper respiratory tract infection. Corticosteroid usage has been associated with central serous choroidoretinopathy, which can cause serous retinal detachments and is thought to be caused by hyperpermeability of the choroidal vessels, combined with alteration in RPE function [7]. However, these are normally restricted to the macula and such extensive retinal detachments are very unlikely to be due to a topically administered steroid. The onset of the disease following creatine usage and the resolution following discontinuation indicates this is the most likely cause. This is the first case report of exudative retinal detachment following creatine supplementation.

\section{Statement of Ethics}

The subject has given written informed consent to publish his case.

\section{Disclosure Statement}

The authors have no relevant disclosures.

\section{Funding Sources}

Not applicable.

\section{Author Contributions}

All authors have been involved with the patient's management and have contributed to the conception, writing, and review of the manuscript. 


\section{Case Reports in Ophthalmology}

\begin{tabular}{l|l}
\hline Case Rep Ophthalmol 2019;10:374-378 \\
\hline DOI: 10.1159/000503853 & $\begin{array}{l}\text { ○ 2019 The Author(s). Published by S. Karger AG, Basel } \\
\text { www.karger.com/cop }\end{array}$ \\
\hline
\end{tabular}

Kopsidas et al.: Bilateral Exudative Retinal Detachment following Creatine Supplementation

\section{References}

1 Davidson AE, Millar ID, Urquhart JE, Burgess-Mullan R, Shweikh Y, Parry N, et al. Missense mutations in a retinal pigment epithelium protein, bestrophin-1, cause retinitis pigmentosa. Am J Hum Genet. 2009 Nov;85(5):581-92.

2 Wallimann T, Tokarska-Schlattner M, Schlattner U. The creatine kinase system and pleiotropic effects of creatine. Amino Acids. 2011 May;40(5):1271-96.

3 de Souza CF, Kalloniatis M, Christie DL, Polkinghorne PJ, McGhee CN, Acosta ML. Creatine transporter immunolocalization in aged human and detached retinas. Invest Ophthalmol Vis Sci. 2012 Apr;53(4):193645.

4 Nakashima T, Tomi M, Katayama K, Tachikawa M, Watanabe M, Terasaki T, et al. Blood-to-retina transport of creatine via creatine transporter (CRT) at the rat inner blood-retinal barrier. J Neurochem. 2004 Jun;89(6):1454-61.

$5 \quad$ Pritchard NR, Kalra PA. Renal dysfunction accompanying oral creatine supplements. Lancet. 1998 Apr;351(9111):1252-3.

6 Strauss O. The Retinal Pigment Epithelium. Kolb H, Fernandez E, Nelson R. Webvision: The Organization of the Retina and Visual System [Internet]. Salt Lake City (UT): University of Utah Health Sciences Center; 2011.

7 Spaide RF, Goldbaum M, Wong DW, Tang KC, Iida T. Serous detachment of the retina. Retina. 2003 Dec;23(6):820-46.
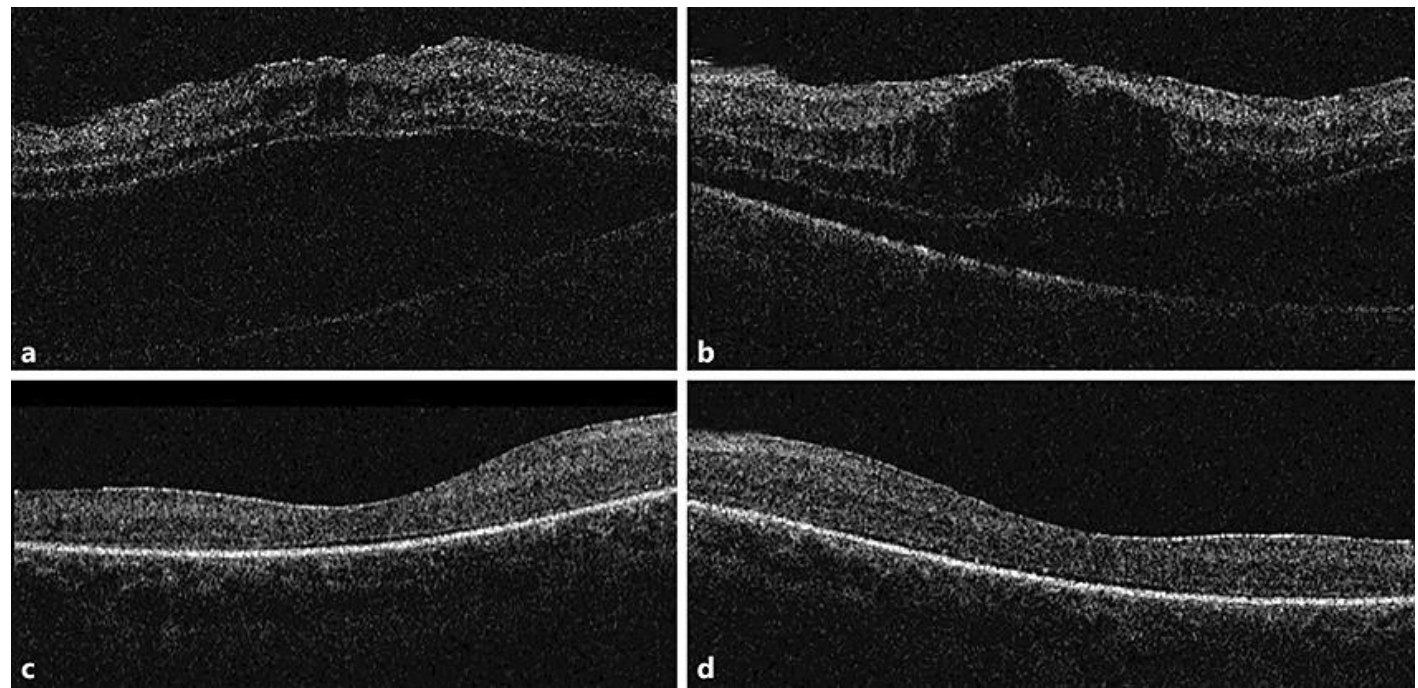

Fig. 1. OCT scan of both eyes at presentation (a right, $\mathbf{b}$ left) and 5 days later (c right, $\mathbf{d}$ left). 


\section{Case Reports in Ophthalmology}

\begin{tabular}{l|l}
\hline Case Rep Ophthalmol 2019;10:374-378 \\
\hline DOI: 10.1159/000503853 & $\begin{array}{l}\text { @ 2019 The Author(s). Published by S. Karger AG, Basel } \\
\text { www.karger.com/cop }\end{array}$ \\
\hline
\end{tabular}

Kopsidas et al.: Bilateral Exudative Retinal Detachment following Creatine Supplementation
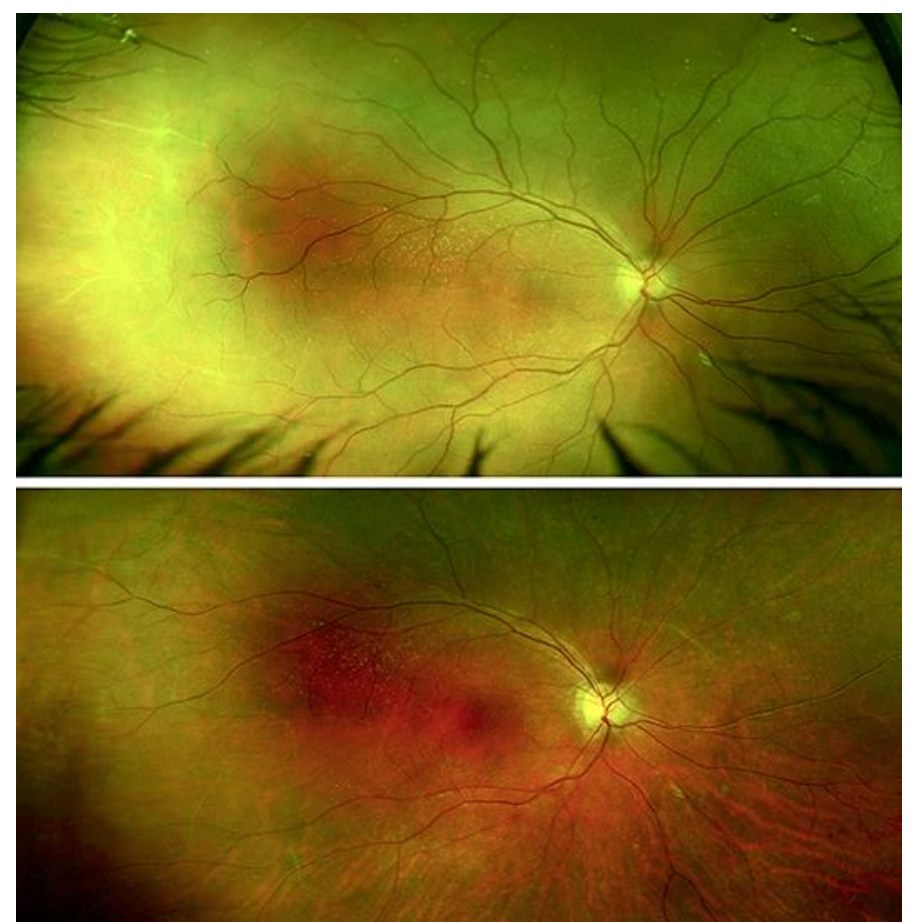

Fig. 2. Wide-field fundus photograph at presentation and 5 days later (right eye).
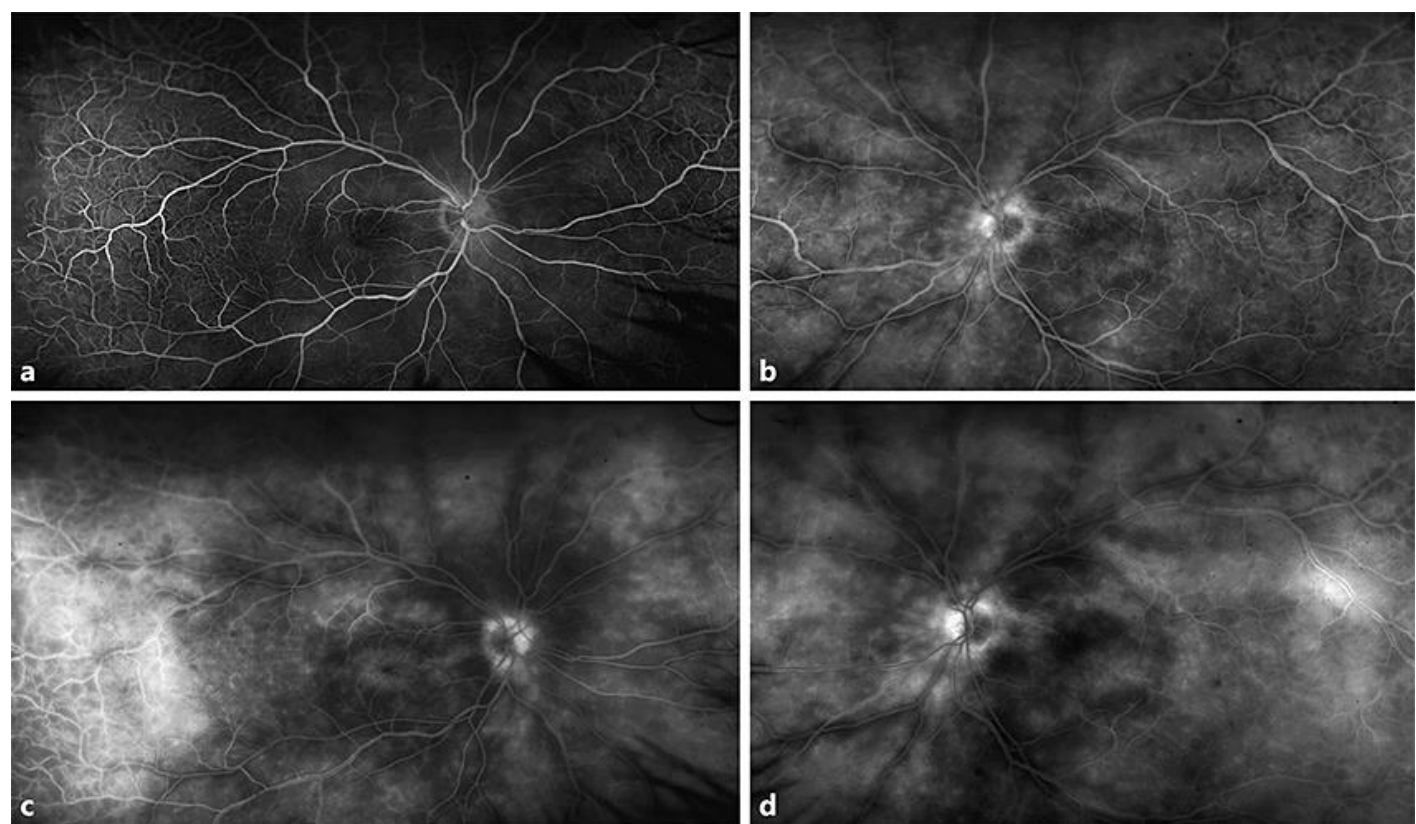

Fig. 3. Fluorescein angiogram of both eyes at presentation (a right early, $\mathbf{b}$ left early, $\mathbf{c}$ right late, $\mathbf{d}$ right late). 